\title{
Simulation of Bistable Laser Diodes with Inhomogeneous Excitation
}

\author{
GANG FANG and TING-WEI TANG * \\ Department of Electrical and Computer Engineering, University of Massachusetts, Amherst, MA 01003
}

\begin{abstract}
A comprehensive time-dependent 1-D computer model is developed for the simulation of multi-section bistable laser diodes. Analysis of a Fabry-Perot (FP) cavity laser diode indicates that the height and shape of the optical input pulse as well as the wavelength play important roles in the set/reset operation of optical switching.
\end{abstract}

Keywords: Transient simulation, bistable laser diodes, optical switching, set/reset operation, undershoot switching

\section{INTRODUCTION}

In recent years, BiStable Laser Diodes (BSLDs) have attracted much attention because of their potential applications in optical switching and wavelength conversion. The simulation of BSLDs involves large-signal analysis, which currently existing LD simulators (such as CLADISS [1]) cannot perform. Most studies of BSLDs are conducted using a set of rate equations that are expressed as a function of time only [2]. Such an approach has two main limitations. First, an approximation of distributed loss is required. Second, the spatial dependence of the gain saturation inside the cavity is not taken into account. Most existing models use a single variable to represent the total photon density without considering the difference in wavelength. Moreover, these models often do not include all pertinent physical processes which compete with each other to influence the dynamic characteristics of the device. Therefore a more sophisticated simulator is needed to assist the design as well as the understanding of the physics of BSLDs.

In this work, a comprehensive time-dependent one-dimensional computer model is developed for the simulation of multi-section bistable laser diodes. Longitudinal spatial hole-burning, nonlinear gain, carrier induced refractive index change (frequency chirp), and thermal effects are rigorously accounted for.

\footnotetext{
*Corresponding author. Fax: 413-545-4611. E-mail: ttang@ecs.umass.edu.
} 


\section{PHYSICAL MODEL}

A typical longitudinal view of a multi-section laser diode is shown in Figure 1. The laser consists of two gain sections and a saturable absorption section in between.

The optical field $\tilde{E}(z, t)$ can be separated into the forward (+) and backward (-) propagating parts, satisfying the traveling-wave rate equations,

$$
\pm \frac{\partial \tilde{E}^{ \pm}}{\partial z}+\frac{1}{v_{g}} \frac{\partial \tilde{E}^{ \pm}}{\partial t}=\left(\frac{2 \pi}{\lambda} n_{r}+i \frac{1}{2} G\right) \tilde{E}^{ \pm}
$$

where

$$
\begin{gathered}
G(\lambda, n(z))=\Gamma g(\lambda, n(z))-\Gamma \alpha_{\mathrm{ac}}-(1-\Gamma) \alpha_{\mathrm{cl}}, \\
n_{r}(\lambda, n(z))=n_{\mathrm{eff}}+\Gamma \Delta n_{r}(\lambda, n(z)),
\end{gathered}
$$

and $\Gamma$ is the confinement factor, $n_{\mathrm{eff}}$ is the effective refractive index determined by waveguide structure, $g$ is the gain function, $\alpha_{\mathrm{ac}}$ and $\alpha_{\mathrm{cl}}$ represent the absorption coefficients in the active layer and cladding layers, respectively, and $\Delta n_{r}$ is the carrier induced change of refractive index.

If we assume $\tilde{E}^{ \pm}(z, t)=E^{ \pm}(z, t) e^{i \phi^{ \pm}(z, t)}$, after substituting into equation (1) and separating the real and imaginary parts, we obtain

$$
\begin{gathered}
\pm \frac{\partial E^{ \pm}}{\partial z}+\frac{1}{v_{g}} \frac{\partial E^{ \pm}}{\partial t}=\frac{1}{2} G(\lambda, n(z)) E^{ \pm} \\
\pm \frac{\partial \phi^{ \pm}}{\partial z}+\frac{1}{v_{g}} \frac{\partial \phi^{ \pm}}{\partial t}=\frac{2 \pi}{\lambda} n_{r}(\lambda, n(z)) .
\end{gathered}
$$

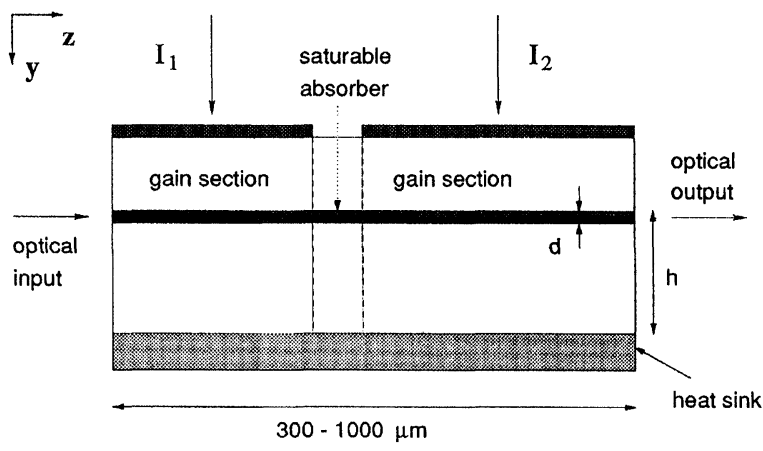

FIGURE 1 Longitudinal structure of a three-section laser diode.
For each longitudinal mode $\left(\lambda_{m}\right)$, the amplitude and phase satisfy the following boundary conditions at $z=0$ and $z=L$ :

$$
\begin{gathered}
E_{m}^{+}(0, t)=r_{1} E_{m}^{-}(0, t), \\
\phi_{m}^{+}(0, t)=\phi_{m}^{-}(0, t)+2 m \pi, \\
E_{m}^{-}(L, t)=r_{2} E_{m}^{+}(L, t), \\
\phi_{m}^{-}(L, t)=\phi_{m}^{+}(L, t),
\end{gathered}
$$

where $m$ is an integer, $r_{1}$ and $r_{2}$ are reflective coefficients of the front and rear facets, respectively. However, for an incident optical field (at $z=0$ ) of an arbitrary wavelength $\left(\lambda_{\text {in }}\right)$, since the incident optical field $t_{1} E_{\text {ex }}(t)$ interferes with the reflected field $r_{1} \tilde{E}^{-}(0, t)$ just inside the left (front) facet, the boundary condition at $z=0$ becomes

$$
\tilde{E}_{\text {in }}^{+}(0, t)=r_{1} \tilde{E}_{\text {in }}^{-}(0, t)+t_{1} E_{\text {ex }}(t),
$$

and at $z=L$, it is given by equations (6) and (7). Under the steady-state condition, equation (3) can be rewritten as

$$
\phi^{-}(0, t)-\phi^{+}(0, t)=\Delta \Phi,
$$

where

$$
\Delta \Phi \equiv \frac{2 \pi}{\lambda} \cdot\left[2 n_{\mathrm{eff}} L+2 \Gamma \int_{0}^{L} \Delta n_{r}(\lambda, n(z)) d z\right]
$$

is the round-trip phase change. For the light in the $m^{\text {th }}$ mode, $\Delta \Phi_{m}$ must be equal to $2 m \pi$. Thus we can calculate wavelength change (frequency chirp) by solving this nonlinear equation. For the incident light, since its wavelength is determined by the external light source, $\Delta \Phi_{\text {in }}$ will change with the carrier density in the active region. Since the round-trip time $\left(2 L / v_{g} \sim 10 p s\right)$ is typically much shorter than the response time $(>0.2 n s)$ for devices under the study, we can still use the boundary condition (8) for the time-dependent problems, instead of solving the rate equations for the phases.

If we define $P_{m}\left(Q_{m}\right)$ as the photon density in $m^{\text {th }}$ mode traveling in the $+z(-z)$ direction, 


$$
\begin{aligned}
& P_{m}=P_{m}(z, t)=\frac{1}{v_{g}}\left|E_{m}^{+}(z, t)\right|^{2} / \hbar \omega_{m} \\
& Q_{m}=Q_{m}(z, t)=\frac{1}{v_{g}}\left|E_{m}^{-}(z, t)\right|^{2} / \hbar \omega_{m}
\end{aligned}
$$

and take into consideration the spontaneous emission, the traveling-wave rate equations for the photon density become,

$$
\begin{aligned}
\frac{\partial P_{m}}{\partial t}+v_{g} \frac{\partial P_{m}}{\partial z}= & v_{g} \cdot G_{m}(n) \cdot P_{m} \\
& +\frac{1}{2} \beta_{\mathrm{sp}} \cdot R_{\mathrm{sp}}(n), \\
\frac{\partial Q_{m}}{\partial t}-v_{g} \frac{\partial Q_{m}}{\partial z}= & v_{g} \cdot G_{m}(n) \cdot Q_{m} \\
& +\frac{1}{2} \beta_{\mathrm{sp}} \cdot R_{\mathrm{sp}}(n),
\end{aligned}
$$

and the corresponding boundary conditions are,

$$
\begin{gathered}
P_{m}(0, t)=R_{1} Q_{m}(0, t), \\
Q_{m}(L, t)=R_{2} P_{m}(L, t),
\end{gathered}
$$

where $R_{1}=\left|r_{1}\right|^{2}$ and $R_{2}=\left|r_{2}\right|^{2}$.

For the incident light $\left(P_{\text {in }}, Q_{\text {in }}\right)$, the rate equations remain the same, while the boundary conditions are different, given by

$$
\begin{aligned}
& \sqrt{P_{\text {in }}(0, t)} \cos \left(\phi_{0}^{+}\right)=\sqrt{R_{1} Q_{\text {in }}(0, t)} \cos \left(\phi_{0}^{-}\right) \\
& +\sqrt{T_{1} P_{\mathrm{ex}}(t),(t)} \\
& \sqrt{P_{\text {in }}(0, t)} \sin \left(\phi_{0}^{+}\right)=\sqrt{R_{1} Q_{\text {in }}(0, t)} \sin \left(\phi_{0}^{-}\right), \\
& \phi_{0}^{-}-\phi_{0}^{+}=\Delta \Phi_{\text {in }}(n), \\
& Q_{\text {in }}(L, t)=R_{2} P_{\text {in }}(L, t),
\end{aligned}
$$

where $\phi_{0}^{+}(t)=\phi_{\text {in }}^{+}(0, t), \phi_{0}^{-}(t)=\phi_{\text {in }}^{-}(0, t), T_{1}=\left|t_{1}\right|^{2}$, and $P_{\mathrm{ex}}(t)=\left(1 / v_{g}\right) E_{\mathrm{ex}}^{2}(t) / \hbar \omega_{\text {in }}$.

We assume that all sections are current controlled and that, due to the leakage, only a fraction $\eta$ of the injected carrier is captured by the active layer where the carriers distribute uniformly in the lateral and transverse directions. No doping is assumed and hence it follows from the charge neutrality condition that the hole density equals the electron density. The rate equation for carrier density $n(z, t)$ in the active layer is given by

$$
\begin{aligned}
\frac{\partial n(z, t)}{\partial t}= & \frac{\eta J(z)}{q d}-\frac{n}{\tau_{\mathrm{nr}}}-R_{\mathrm{sp}}(n)-C_{\text {Aug }} n^{3} \\
& -v_{g} \Gamma \cdot\left\{\sum_{m} g_{m}(n)\left(P_{m}+Q_{m}\right)\right. \\
& \left.+g_{\text {in }}(n)\left(P_{\text {in }}+Q_{\text {in }}\right)\right\} .
\end{aligned}
$$

In our model, the linear gain is approximated by

$g_{L}(\lambda, n)= \begin{cases}g_{A} \cdot\left(n-n_{0}\right), & n<n_{10} \\ g_{2} \cdot n^{2}+g_{1} \cdot n+g_{0}, & n_{20}>n>n_{10} \\ g_{G} \cdot\left(n-n_{0}\right) . & n>n_{20}\end{cases}$

where $g_{A}$ and $g_{G}$ are the differential gain coefficients in the gain and loss region, $n_{0}$ is the transparency carrier density. These parameters can be determined by theoretical calculation or experiment. It should be emphasized that different values of $g_{A}, g_{G}$, and $n_{0}$ are used for each longitudinal mode and optical input.

The stimulated emission becomes nonlinear whenever large optical intensities exist in the active layer. To account for this effect a simplified gain model is used, i.e.,

$$
g_{N L}(\lambda, n)=\left[1-\varepsilon_{N L} \sum_{m, \text { in }}(P+Q)\right] \cdot g_{L}(\lambda, n)
$$

where $\varepsilon_{\mathrm{NL}}$ is the material gain compression factor. Then the carrier-induced change of refractive index is assumed to be given by

$$
\Delta n_{r}(\lambda, n)=-\alpha_{R} \frac{g_{L}(\lambda, n)}{2 k},
$$

where $\alpha_{R}$ is assumed to be a constant. 


\section{NUMERICAL SOLUTION METHODS}

In this work, we have improved the spatial discretization scheme described in [3]. A first-order backward finite difference formula is applied to $P(z, t)$ and a forward difference applied to $Q(z, t)$. The photon density is defined at the nodes while the carrier density is defined between two adjacent nodes. The change in the carrier density in a region now depends on the average number of photons surrounding that region. A trapezoidal rule/backward-differentiation-formula composite method [4] has been implemented for the time integration. The system of discretized nonlinear equations is solved simultaneously using the full Newton method. The simulation result shown in Figure 4 can be obtained within 20 minutes (CPU time) on a DECstation 5000/200.

\section{SIMULATION RESULTS}

A two-electrode FP cavity InGaAs/InP laser diode [5] has been simulated. An example of set-reset operation (undershoot switching) using a single wavelength $\left(\lambda_{\text {in }}=1557.6 \mathrm{~nm}\right)$ light is shown in Figure 2. The bias current is chosen to be $I_{1}=$ $18.4 \mathrm{~mA}$. The intensities of pulses for set and reset are $0.874 \mathrm{~mW}$ and $13.12 \mathrm{~mW}$, respectively.

The mechanism of undershoot switching is believed to be dependent on different changing rates of carrier densities in the gain and absorption regions under a variation of the optical excitation. We have found that the shape of the pulses plays an important role in undershoot switching, as shown in Figures 3 and 4.

From our simulation results, a subnanosecond switch-on (rise) time is observed, while the switchoff (fall) time of the device is about $3 \mathrm{~ns}$. We also found that an input pulse with a higher intensity and faster fall time would reduce the switch-off time somewhat but still no less than 2 ns.

The wavelength of the injected optical pulses is also crucial for the undershoot switching. The minimum set/reset powers will be modulated by
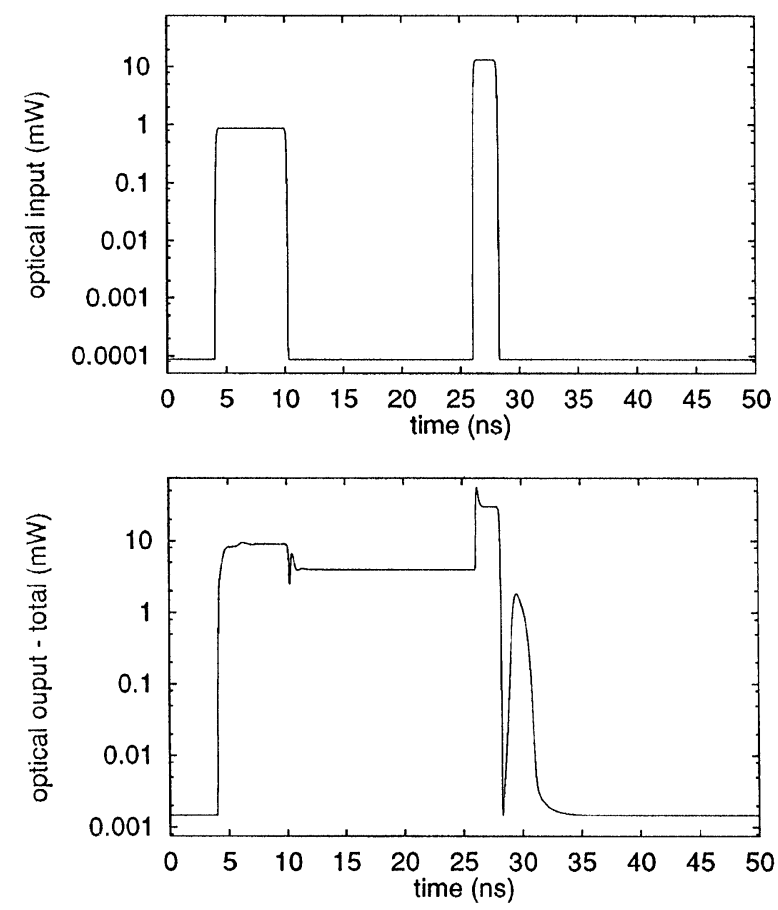

FIGURE 2 The set-reset operation (undershoot shooting): optical input and optical output.

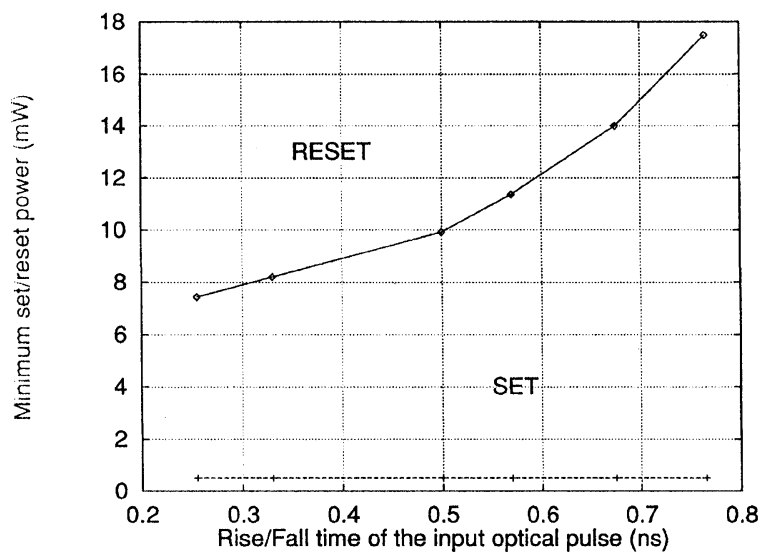

FIGURE 3 Influence of the shape (rise/fall time) of the input optical pulses.

the resonant characteristic of the Fabry-Perot cavity as shown in Figure 5. The two curves are almost parallel. Both have minima near the resonant wavelength. 

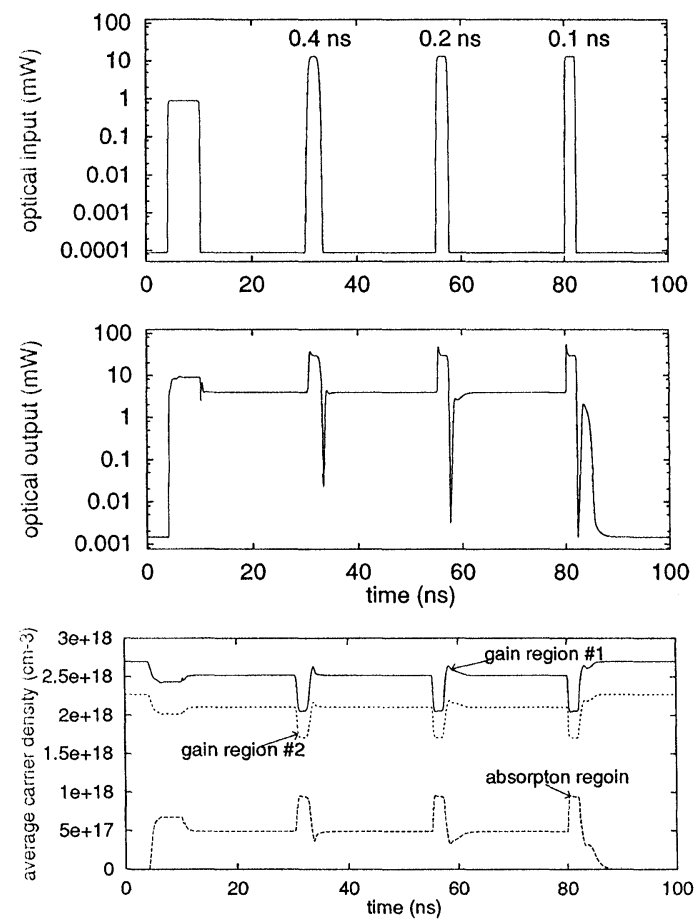

FIGURE 4 Examples of the set-reset operation: the effects of pulse shape on undershooting for $W_{\text {in }}=13.12 \mathrm{~mW}, \lambda_{\text {in }}=$ $1557.6 \mathrm{~nm}$.

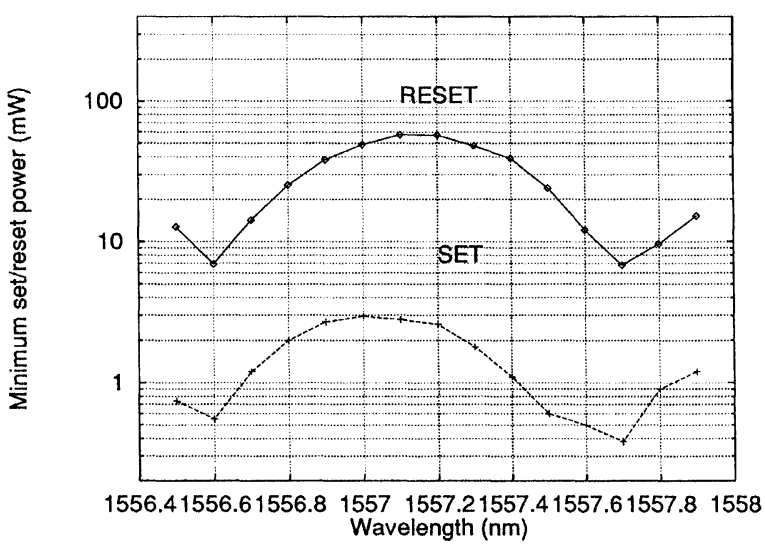

FIGURE 5 Influence of the wavelength of the optical signals on the set-reset operation.

\section{CONCLUSION}

In this paper, a comprehensive transient 1-D computer model for the simulation of multi- section bistable laser diodes is presented. Traveling wave rate equations are solved for each longitudinal mode and optical input. All the important effects have been included in this model.

Analyses of two-electrode FP cavity InGaAsP/ InP laser diodes incorporating an absorber region are carried out to illustrate the capability of the simulator. Special attention is paid to the optical set-reset operation (undershoot switching). Both the height and shape (rise/fall time) as well as the wavelength of the optical input pulse are shown to play an important roles in undershoot switching.

\section{References}

[1] Vankwikelberge, P., Morthier, G. and Baets, R. (1990). "CLADISS, a longitudinal, multimode model for the analysis of the static, dynamic and stochastic behaveiour of diode lasers with distributed feedback", IEEE $J$. Quantum Electron., 26, 1728-1741.

[2] Kawaguchi, H. (1987). "Progress in optical functional devices using two-section laser diodes/amplifiers" Optical and Quantum Electron., 19, S1-S36.

[3] Wong, Y. L. and Carroll, J. E. (1987). “A travelling-wave Rate Equation Analysis For Semiconductor Laser", SolidState Electron., 30, 13-19.

[4] Bank, B. E., Coughan, W. M., Fichtner, W., Grosse, E. H., Rose, D. J. and Smith, R. K. (1985). "Transient Simulation of Silicon Devices and Circuit", IEEE Trans. on CAD/ ICAS, CAD-4, 436-451.

[5] Okada, M., Takizawa, H., Kikuchi, H. and Fujikake, H. (1993). "The effect of a Detuned Optical Input on Bistable Laser Diodes with Inhomogeneous Current Injection", IEEE J Quantum Electron., 29, 109-119.

\section{Authors' Biographies}

Gang Fang received his M.S.E.C.E. from University of Massachusetts. He is now working on his doctoral degree at Carnegie Mellon University. His research interests include physical layout automation, circuit analysis, and technology CAD.

Ting-wei Tang is Professor of Electrical and Computer Engineering at University of Massachusetts, Amherst. His research interests include semiconductor device physics and numerical simulation. 

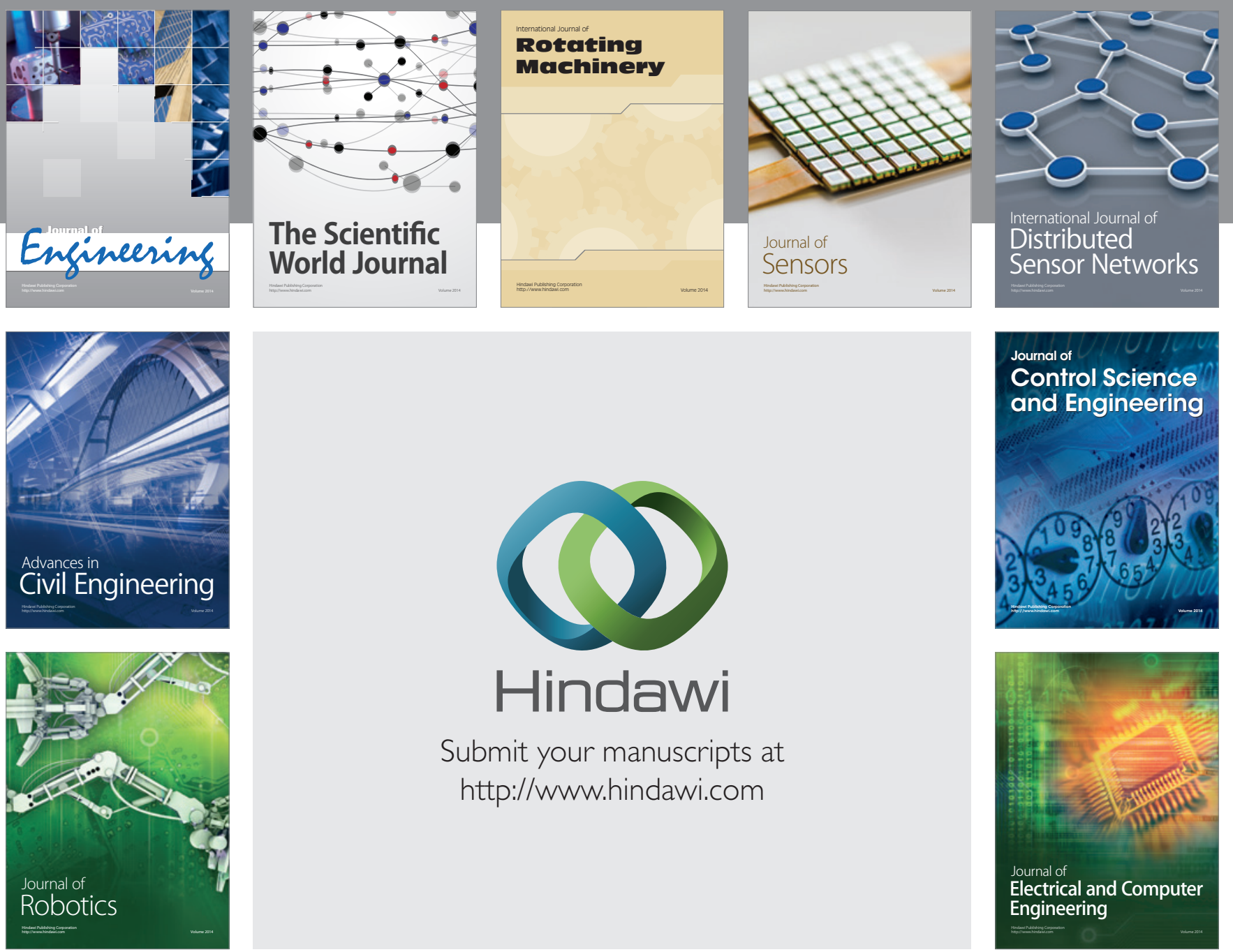

Submit your manuscripts at

http://www.hindawi.com
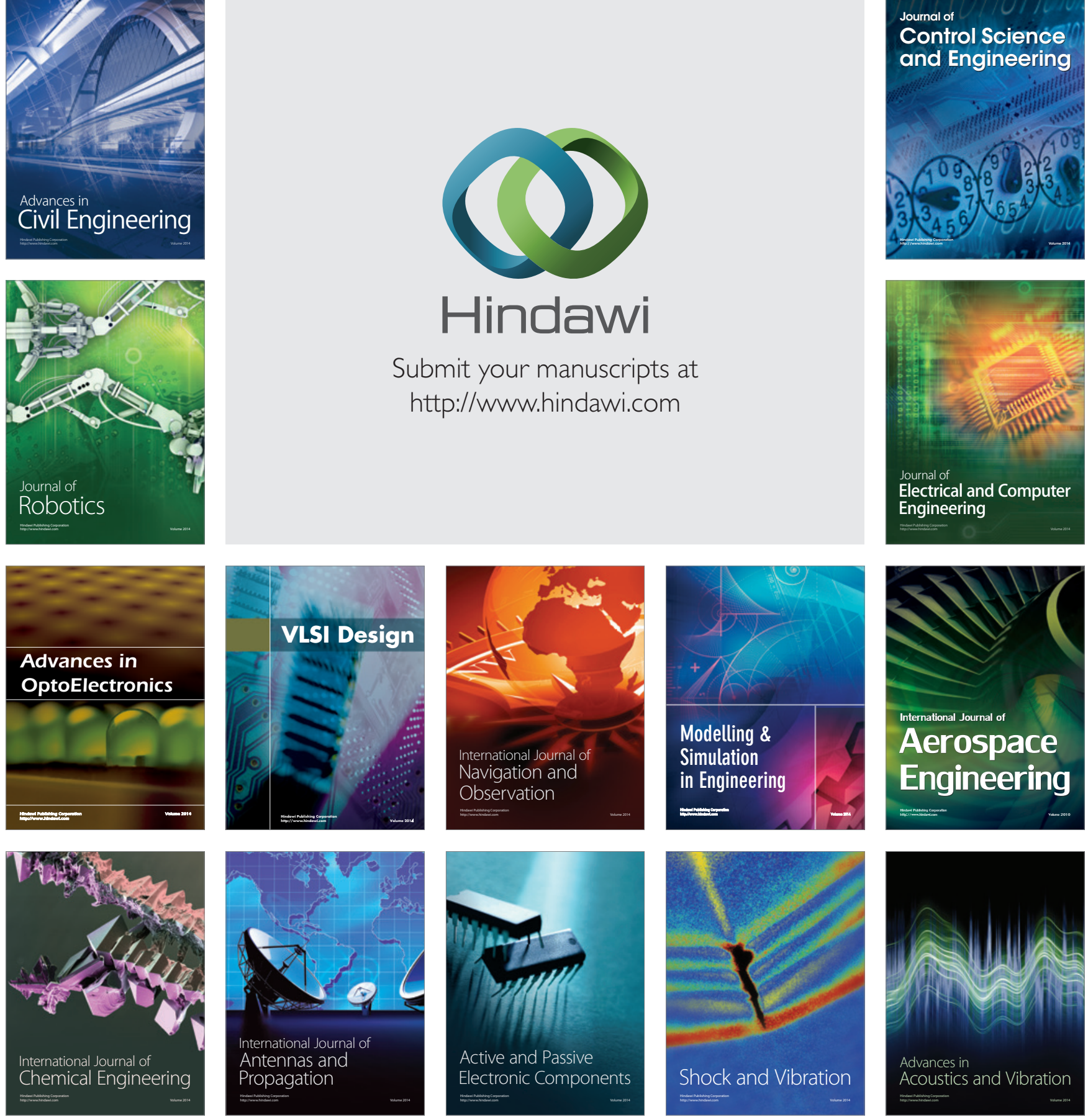